\title{
Annual and seasonal variations in the low-latitude topside ionosphere
}

\author{
Y. Z. Su${ }^{1}$, G. J. Bailey ${ }^{1}$, K.-I. Oyama ${ }^{2}$ \\ ${ }^{1}$ School of Mathematics and Statistics, Applied Mathematics Section, The University of Sheffield, Sheffield S3 7RH, UK \\ Fax: +44 11422 23739; e-mail: Y.Su@sheffield.ac.uk \\ ${ }^{2}$ Institute of Space and Astronautical Science, 3-1-1 Yoshinodai, Sagamihara, Kanagawa 229, Japan
}

Received: 22 October 1997 / Revised: 27 February 1998 / Accepted: 2 March 1998

\begin{abstract}
Annual and seasonal variations in the lowlatitude topside ionosphere are investigated using observations made by the Hinotori satellite and the Sheffield University Plasmasphere Ionosphere Model (SUPIM). The observed electron densities at $600 \mathrm{~km}$ altitude show a strong annual anomaly at all longitudes. The average electron densities of conjugate latitudes within the latitude range $\pm 25^{\circ}$ are higher at the December solstice than at the June solstice by about $100 \%$ during daytime and $30 \%$ during night-time. Model calculations show that the annual variations in the neutral gas densities play important roles. The model values obtained from calculations with inputs for the neutral densities obtained from MSIS86 reproduce the general behaviour of the observed annual anomaly. However, the differences in the modelled electron densities at the two solstices are only about $30 \%$ of that seen in the observed values. The model calculations suggest that while the differences between the solstice values of neutral wind, resulting from the coupling of the neutral gas and plasma, may also make a significant contribution to the daytime annual anomaly, the $\mathbf{E} \times \mathbf{B}$ drift velocity may slightly weaken the annual anomaly during daytime and strengthen the anomaly during the post-sunset period. It is suggested that energy sources, other than those arising from the $6 \%$ difference in the solar EUV fluxes at the two solstices due to the change in the Sun-Earth distance, may contribute to the annual anomaly. Observations show strong seasonal variations at the solstices, with the electron density at 600 $\mathrm{km}$ altitude being higher in the summer hemisphere than in the winter hemisphere, contrary to the behaviour in NmF2. Model calculations confirm that the seasonal behaviour results from effects caused by transequatorial component of the neutral wind in the direction summer hemisphere to winter hemisphere.
\end{abstract}

\section{Introduction}

Yearly variations of the Earth's ionosphere can be represented by a superposition of annual, seasonal and semi-annual components and the yearly average. While the cycle of the semi-annual component is 6 months, the cycles of both the annual and seasonal components are 12 months. The difference between the annual and seasonal components is that the annual component has the same phase in both hemispheres whilst the seasonal components of each hemisphere are 6 months out of phase. Both the annual and seasonal components have their maxima and minima around the June and December solstices.

It was found by Yonezawa (1959), from averages of observations made at conjugate stations, that the F2layer peak electron density $(\mathrm{NmF} 2)$ at the December solstice exceeds that at the June solstice by around $20 \%$. This feature of the annual component is known as the annual anomaly. Yonezawa (1971) extended his earlier work by examining the latitudinal and solar cycle dependencies. His results showed that, in general, the relative amplitude of the annual component in NmF2 decreases slowly with increasing latitude and solar activity during daytime and is smaller at night compared to daytime. The seasonal component in the $\mathrm{F}$ region is characterized by the winter anomaly, i.e. $\mathrm{NmF} 2$ is higher in winter than in summer (Rishbeth and Setty, 1961). Using data from 140 stations, Torr and Torr (1973) have investigated the differences in NmF2 between the December and June solstices; the annual and seasonal components were not separated. Torr and Torr found $\mathrm{NmF} 2$ to be, in general, higher in winter than in summer in both the northern and southern hemispheres and that the differences in $\mathrm{NmF} 2$ at the two solstices are stronger in the northern hemisphere than in the southern hemisphere; the differences are small at equatorial latitudes. These results imply that there are winter and annual anomalies in $\mathrm{NmF} 2$ and that the seasonal anomaly predominates over the annual anomaly at most locations. 
Titheridge and Buonsanto (1983) have compared the values of total electron content (TEC) measured at conjugate sites at $\pm 20^{\circ}$ latitude and non-conjugate sites near $\pm 35^{\circ}$ latitude. They found that TEC is larger around the December solstice than around the June solstice in both the northern and southern hemispheres. This result suggests that, for TEC, the annual anomaly predominates over the seasonal anomaly.

The winter anomaly has been explained successfully by changes in thermospheric composition, the atomic/ molecular ratio in winter is higher than in summer (e.g. Rishbeth and Setty, 1961; Rüster and King, 1973), and from global thermospheric circulation (e.g. Millward et al., 1996a). The annual anomaly is dependent upon many parameters. The annual and seasonal variations of the topside ionosphere have yet to be investigated in detail. Observational evidence indicates that the behaviour in the topside ionosphere is rather different to that in the $\mathrm{F}$ region. For example, there is no winter anomaly in the topside ionosphere whereas there is in the $\mathrm{F}$ region (e.g. Torr and Torr, 1973).

In the present study, data collected from more than 600 orbits of the Hinotori satellite have been used to investigate the global behaviour of the annual and seasonal variations in the topside ionosphere at low latitudes. The annual and seasonal components are determined by comparing observations made at both solstices at conjugate locations. An analysis of the observations shows that there is a very large annual component in the topside ionosphere; the global average electron density during daytime at $600 \mathrm{~km}$ altitude at the December solstice being $100 \%$ higher than that at the June solstice. This result is contrary to expectations, since the difference in the solar EUV flux between the December and June solstices, due to the changes in the Sun-Earth distance, is only about $6 \%$. Suggested source mechanisms for the annual and seasonal variations in the topside ionosphere at low latitudes are investigated by using SUPIM (Sheffield University plasmasphere ionosphere model) (Bailey and Sellek, 1990; Bailey et al., 1993, 1997).

\section{Observations}

The Hinotori satellite was launched into a near-circular orbit at $600 \mathrm{~km}$ altitude with an inclination of $31^{\circ}$ in February 1981 and it operated until June 1982. The orbital duration of the satellite was about $90 \mathrm{~min}$. Since the memory of the recorder onboard the satellite was limited, only data from about one orbit could be stored at any one time. The data were transmitted to the data acquisition station at the Kagoshima Space Centre (KSC, $\left.131{ }^{\circ} \mathrm{E}\right)$ before the satellite passed the station. Thus, data were transmitted only from those orbits which passed KSC, i.e. from 4 to 6 orbits per day (Watanabe and Oya, 1986). The electron density was measured by an impedance probe in an upper resonance mode (Watanabe and Oya, 1986). The relative error of the electron density measurements is about $10 \%$. The electron density data used in the present study were made during the 1981 June solstice period and during the 1981-1982 December solstice period on days when $A p \leq 30$. The solstice periods are defined by the two 91 days centred on the June and December solstices. This criterion was satisfied on 67 days and 327 orbits for the June solstice period and on 58 days and 313 orbits for the December solstice period. The average value of F10.7 for the selected days was 178 for the June solstice period and 189 for the December solstice period; the difference in F10.7 between the two solstice periods is $6 \%$. For each solstice period, the data were averaged into bins of $1 \mathrm{~h}$ in local time and $5^{\circ}$ in magnetic latitude. The results are shown in Fig. 1a-c.

It can be seen from Fig. $1 \mathrm{a}-\mathrm{c}$ that there is a superposition of the annual and seasonal components in the differences of the average electron density for the two solstice periods. For each period, the average electron density at $600 \mathrm{~km}$ altitude is higher in the summer hemisphere (northern hemisphere for the June solstice and southern hemisphere for the December solstice) than in the winter hemisphere. This feature is contrary to the behaviour of the winter anomaly in the $\mathrm{F}$ region. Around local noon, the asymmetries in the electron density with respect to the magnetic equator are stronger at the December solstice than at the June solstice. Because of the existence of a large annual component, the electron densities in the southern hemisphere are much higher during summer (December solstice) than during winter (June solstice). In the northern hemisphere, on the other hand, the electron densities during summer (June solstice) are slightly higher at the higher latitudes or lower at all latitudes. At the magnetic equator, the electron density at the December solstice is about a factor of 2 greater than that at the June solstice.

To give a quantitative description of the annual anomaly, the electron densities shown in Fig. 1 have been averaged for conjugate locations at $5^{\circ}$ intervals for each solstice; the purpose of taking averages is to remove seasonal effects. The diurnal variations of the electron densities at the equator and the values averaged for latitudes $\pm 5^{\circ}, \pm 10^{\circ}, \pm 15^{\circ}, \pm 20^{\circ}$ and $\pm 25^{\circ}$ are shown in Fig. 2. It can be seen from Fig. 2 that during daytime the average electron densities are much higher at the December solstice than at the June solstice. For each of the latitudes under consideration, the daytime values of electron density at the December solstice are higher than at the June solstice by about $5 \times 10^{5} \mathrm{~cm}^{-3}$, i.e. higher by between 70 and $175 \%$. There is an evening enhancement at the December solstice. Thus, as the evening enhancement is absent at the June solstice, the annual anomaly is still strong after sunset. The annual anomaly becomes smaller during the post-sunset period. The differences in electron density are smallest, by about $5 \times 10^{4} \mathrm{~cm}^{-3}$, i.e. $30 \%$ on average, during the postmidnight period.

To investigate the longitudinal effects, we have separated the data into six longitude sectors and calculated the latitudinal variations of the average daytime (12:00-18:00 LT) and night-time (01:00-06:00 LT) electron densities. It can be seen from Fig. 1 that 

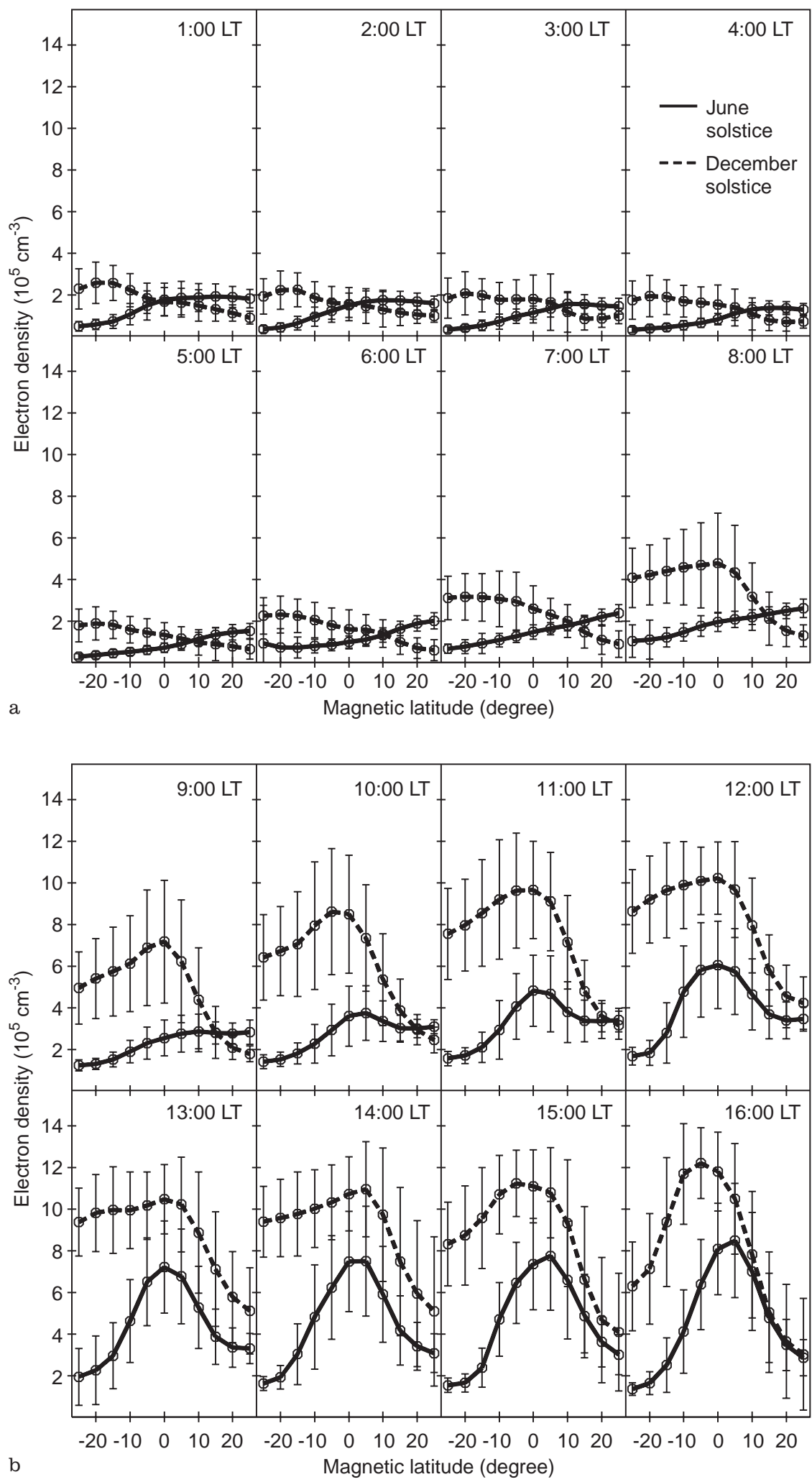

Fig. 1a-c. Latitudinal variations of the average observed electron density for June solstice (solid curves) and December solstice (dashed curves) a at 01:00-08:00 LT. b 09:00-16:00 LT, c 17:00-24:00 LT within each time-period the latitude profiles vary little with time. The choice of longitudes for each longitude sector are based on the configuration of the geomagnetic field, see $\mathrm{Su}$ et al. (1996) for details. The latitudinal coverage of the data is incomplete in some longitude sectors owing to the operating mode of the satellite.
Figure $3 \mathrm{a}$ and $\mathrm{b}$ shows the latitudinal variations of the daytime and night-time averaged electron densities for each longitude sector. Although there are longitudinal differences, it can be seen for each longitude sector that the general features in the annual and seasonal variations are similar to those shown in Fig. 1. This result 


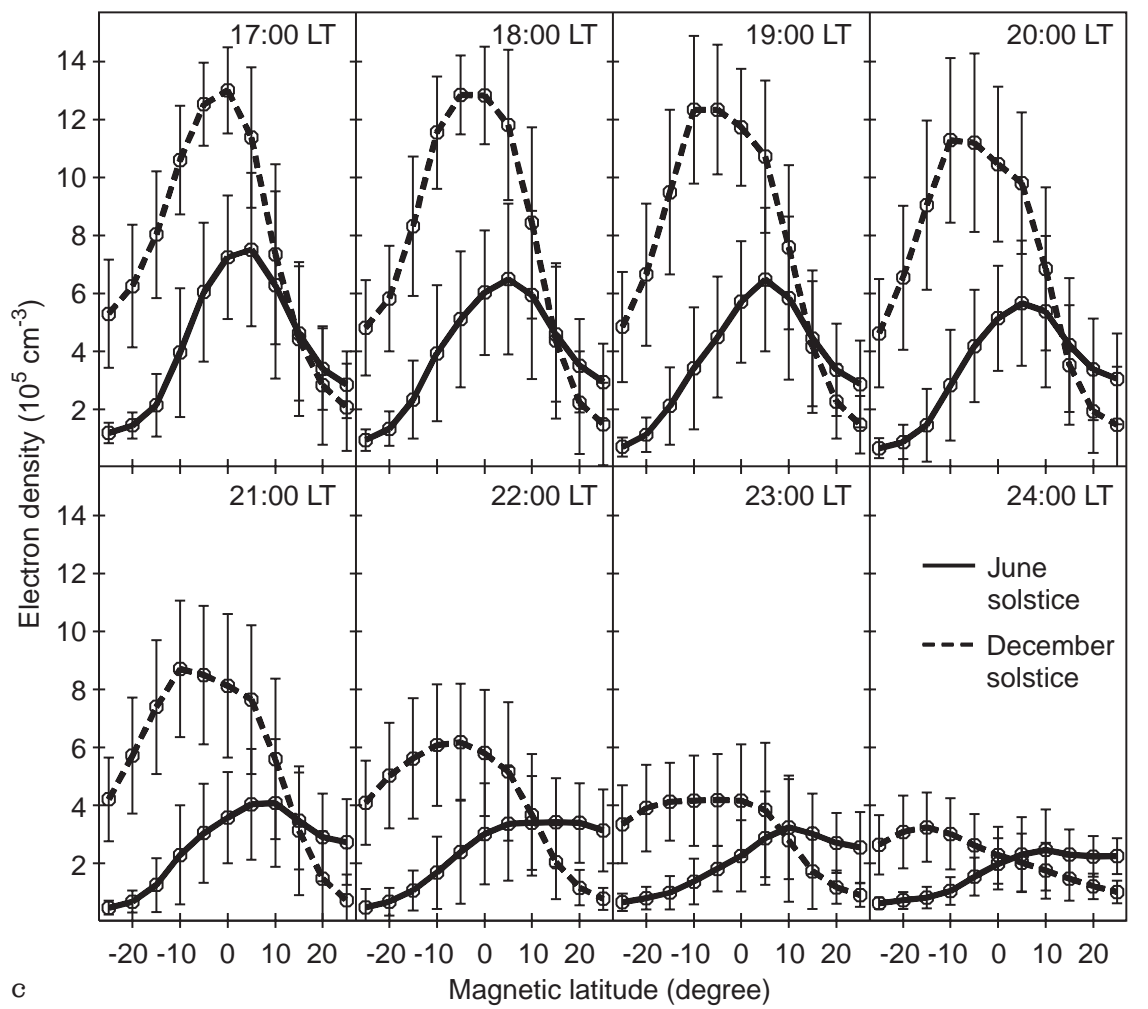

Fig. 1c.
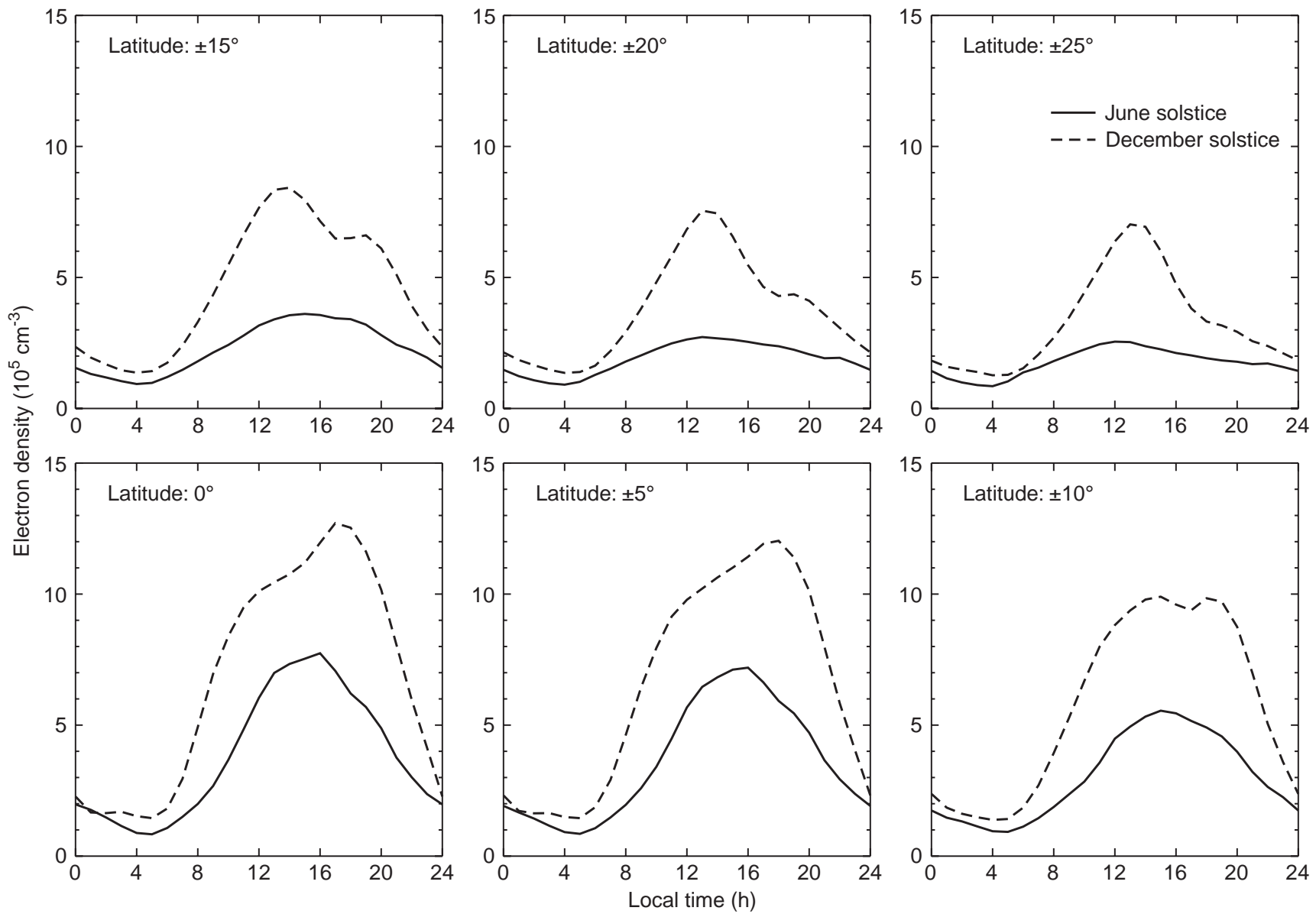

Fig. 2. Diurnal variations of the observed electron density at latitude $0^{\circ}$, and the averages of the observed electron densities at latitudes $\pm 5^{\circ}$, $\pm 10^{\circ}, \pm 15^{\circ}, \pm 20^{\circ}$ and $\pm 25^{\circ}$, for June solstice (solid curves) and December solstice (dashed curves) 
indicates that the annual anomaly and the seasonal variations shown in Fig. 1 have global behaviour.

\section{Model calculations and results}

The model used for the present study is SUPIM, the Sheffield University Plasmasphere Ionosphere Model (Bailey and Sellek, 1990; Bailey et al., 1993, 1997). In the model, coupled time-dependent equations of continuity, momentum and energy balance for the $\mathrm{O}^{+}, \mathrm{H}^{+}, \mathrm{He}^{+}$, $\mathrm{N}_{2}^{+}, \mathrm{O}_{2}^{+}$and $\mathrm{NO}^{+}$ions and the electrons are solved along closed magnetic field lines for the ion and electron densities, field-aligned velocities and temperatures. In the version of the model used for the present study, the $\mathrm{O}^{+}$ion is separated into its $4 \mathrm{~S}, 2 \mathrm{D}$ and $2 \mathrm{P}$ states $(\mathrm{Su}$ et al., 1996). Vibrationally excited $\mathrm{N}_{2}$ is also included in the model. It is taken into account by modifying the chemical reaction rate for $\mathrm{O}^{+}$with $\mathrm{N}_{2}$ by the first-order approximation described in Buonsanto (1995) and $\mathrm{Su}$ et al. (1997). Neither of these two enhancements to SUPIM have significant effects on the results of the present study. The solar extreme ultraviolet (EUV) fluxes are obtained from the EUV94 model (Tobiska, private communication); a revised version of the EUV91 model (Tobiska, 1991). The concentrations and temperatures of the neutral gases are obtained from the MSIS86 thermosphere model (Hedin, 1987). Two neutral wind models are used in the study, the HWM90 thermospheric wind model (Hedin et al., 1991) and a model based on the observed values of electron density.

There is observational and theoretical evidence that the equatorial vertical $\mathbf{E} \times \mathbf{B}$ drift velocity varies with altitude (Pingree and Fejer, 1987; Murphy and Heelis, 1986; Su et al., 1995). This variation is taken into account in the model calculations by using two equatorial vertical drift velocity models, one for field lines with apex altitude less than $400 \mathrm{~km}$ and a second for field lines with apex altitude greater than $1800 \mathrm{~km}$; linear interpolation is used for the field lines with intermediate apex altitudes. Except for one set of calculations, carried out to study the effects of $\mathbf{E} \times \mathbf{B}$ drift, the same drift velocity models have been used for both the December solstice and the June solstice. For the standard $\mathbf{E} \times \mathbf{B}$ drift model, the drift used for the low apex-altitude field lines is based on observations made by the Jicamarca radar (Fejer et al., 1991) and for the high apex-altitude field lines on observations made by the Arecibo radar (Fejer, 1993). Both sets of observations were made during the December solstice period. The longitudinal variations of the $\mathbf{E} \times \mathbf{B}$ drift velocity are small (Fejer et al., 1995) and are not considered.

The model calculations have been carried out for magnetically quiet $(A p=5)$ conditions at the June (day 172) and December (day 356) solstices. The same values of F10.7 (177) and solar EUV fluxes have been used in the model calculations for both solstices to avoid differences in the model values that would arise through the effects of solar activity changes. As the observed values are averaged over all longitudes, an axial-centred dipole representation (which is independent of longitude) is used for the geomagnetic field in the model cal- culations. The model calculations were carried out for several magnetic field lines arranged to provide reasonable 24-h coverage of the model values at $600 \mathrm{~km}$ altitude within the specified latitude region $\left( \pm 25^{\circ}\right)$. For the present calculations, the apex altitudes of the field lines, before the onset of $\mathbf{E} \times \mathbf{B}$ drift, range from 150 to $5500 \mathrm{~km}$. Details of the numerical procedure are provided in Bailey and Balan (1996).

The modelled electron densities at $600 \mathrm{~km}$ altitude, for both the June and December solstices, from calculations which use the HWM90 neutral wind model and the standard $\mathbf{E} \times \mathbf{B}$ drift model are shown in Fig. 4 by the solid and long-dashed curves, respectively. The use of the same drift model for both solstices removes the differences that may arise from the use of different drifts.

We first discuss the seasonal variations. The model results show for each solstice that the modelled electron densities at $600 \mathrm{~km}$ altitude are much higher in the summer hemisphere than in the winter hemisphere. This feature is caused by the neutral wind. The neutral wind has a strong transequatorial component blowing from the summer hemisphere to the winter hemisphere, i.e. a southward component at the June solstice and a northward component at the December solstice. Figure 5 shows the neutral wind pattern given by HWM90 for the June solstice used as model input. The transequatorial component of the neutral wind moves the $F$ region downwards/upwards in the winter/summer hemisphere so that the electron density decreases/increases in the winter/summer hemisphere. The electron densities derived from the model calculations with zero neutral wind, results not shown, are almost symmetric about the magnetic equator.

The results obtained from the model when the neutral wind is given by HWM90 reproduce the observations that the electron density at $600 \mathrm{~km}$ altitude is higher in the summer hemisphere than in the winter hemisphere at both the December and June solstices. However, the asymmetries in the modelled values, with respect to the magnetic equator, are much stronger than shown by the observations. This result suggests that the transequatorial components of the neutral wind in the direction summer hemisphere to winter hemisphere, as predicted by HWM90, are too large. To improve the agreement between the modelled and observed values, model calculations have been carried out using a revised neutral wind model. In the revised wind model, the wind components that blow from the summer hemisphere to the winter hemisphere (southward component in June solstice and northward component in December solstice), as given by HWM90, are reduced by $30 \mathrm{~m} \mathrm{~s}^{-1}$ between 08:00 LT and 13:00 LT and by $15 \mathrm{~m} \mathrm{~s}^{-1}$ at other local times. The results obtained for each of the two solstices from calculations that use the revised winds are also displayed in Fig. 4. It can be seen that the reduction in the summer-hemisphere to winter-hemisphere components of the neutral wind results in smaller asymmetries in the electron density at $600 \mathrm{~km}$ altitude. These modelled values are in better agreement with the observations. The model results suggest, for the solstices, that a transequatorial wind blowing from the summer 

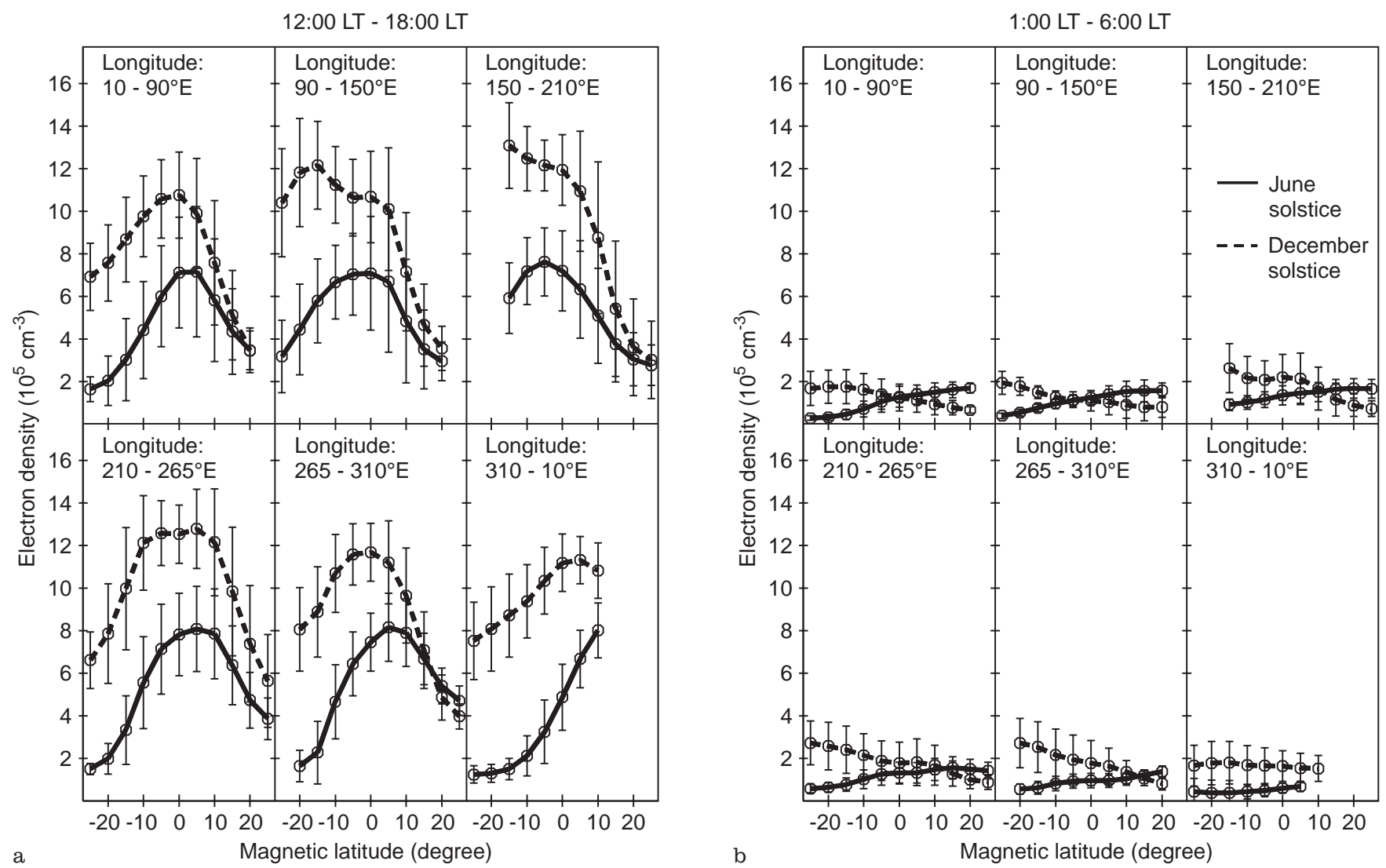

Fig. 3. Latitudinal variations of the average a daytime (12:00-18:00 LT), b night-time (01:00-06:00 LT) observed electron density in six longitude sectors. The longitudinal range of each sector is shown at the top of each panel

hemisphere to the winter hemisphere is responsible for the electron densities at $600 \mathrm{~km}$ altitude being higher in the summer hemisphere than in the winter hemisphere and that the transequatorial component of the wind given by HWM90 is too large. These suggestions are in accordance with the suggestions made in our previous study (Su et al., 1995). The fact that the asymmetries in the observed electron densities around local noon (see Fig. 1) are stronger at the December solstice than at the June solstice indicates that the wind component blowing from the summer hemisphere to the winter hemisphere is stronger at the December solstice than at the June solstice. Our model results do not support the suggestion of Torr and Torr (1973) that the higher electron densities in the summer topside ionosphere, compared to winter, is due to the scale height being larger as a result of the plasma temperature being higher in summer. In fact, both the observed and modelled values ( $\mathrm{Su}$ et al., 1995) show that the electron temperature is lower in the summer hemisphere than in the winter hemisphere. It can be seen from Fig. 4 that, for both wind models, there is an annual component in the modelled electron densities with higher values at the December solstice.

For the remainder of this section we concentrate our attention on the results obtained from the calculations that have used the revised neutral wind model, since the model results for the revised neutral wind model are in better agreement with observations than the results for the HWM90 wind model.

The diurnal variations of the electron densities at the equator and the averages of the values at the conjugate latitudes $\pm 5^{\circ}, \pm 10^{\circ}, \pm 15^{\circ}, \pm 20^{\circ}$ and $\pm 25^{\circ}$, from calculations that use the revised neutral wind model, are displayed in Fig. 6. This figure shows that for each of the latitudes under consideration, the average densities are higher at the December solstice than at the June solstice and that the differences are larger during daytime than during night-time; this feature is in accordance with the observations shown in Fig. 2. However, the magnitude of the annual anomaly seen in the modelled values is much smaller than that seen in the observations; the modelled daytime difference in the electron density at the two solstices is about $30 \%$, but the difference in the observed values is about $100 \%$. The neutral wind is not responsible for the annual anomaly, as the model results from calculations with zero neutral wind (figure not shown) show percentage differences in the electron densities of the two solstices to be similar to those shown in Fig. 6.

We now investigate the effects of changes in neutral composition on the annual variation of the electron density at $600 \mathrm{~km}$ altitude. The diurnal variations of the average neutral gas densities for $\mathrm{O}, \mathrm{N}_{2}$ and $\mathrm{O}_{2}$ over the 


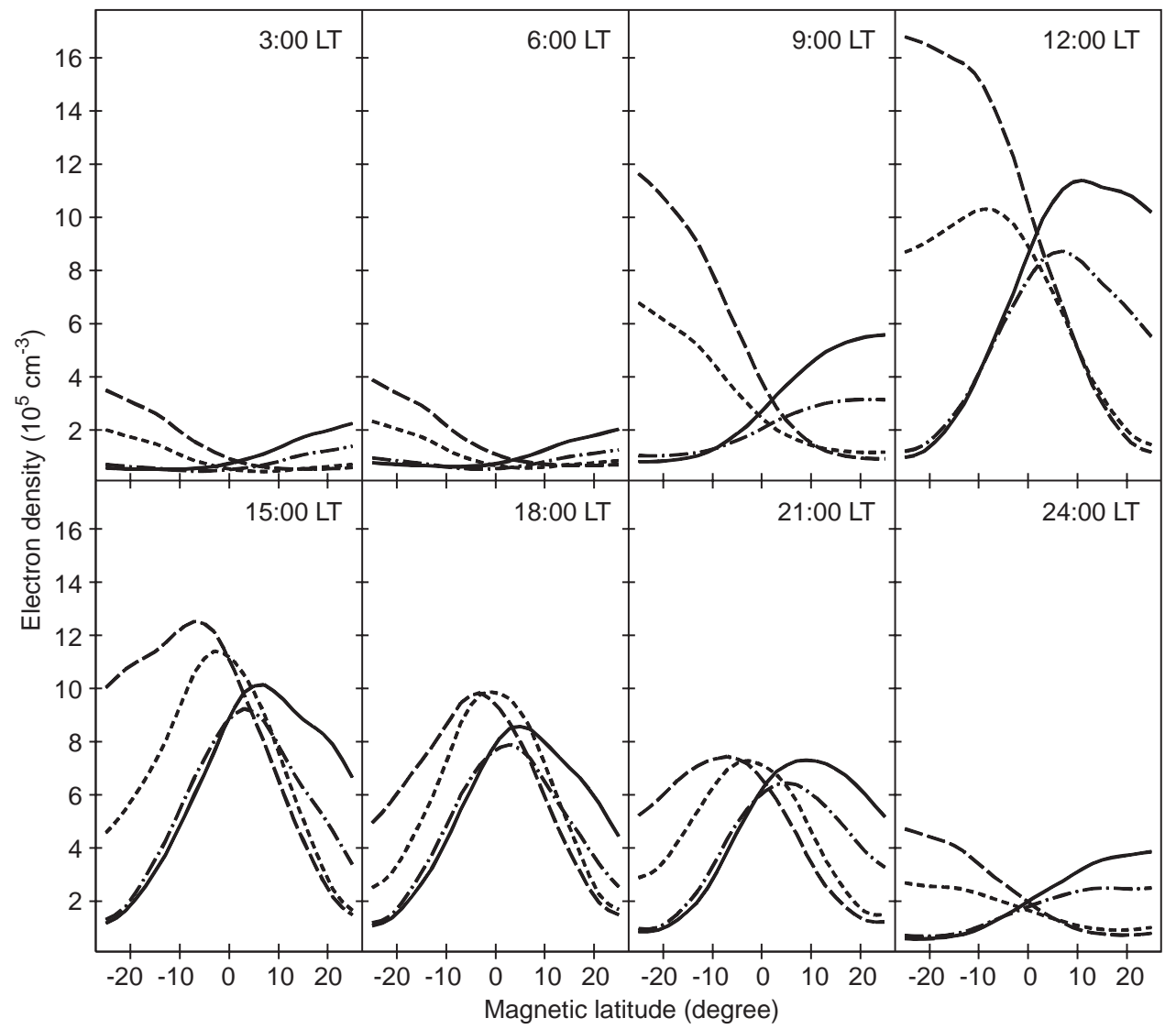

Fig. 4. Latitudinal variations of the modelled electron density, at intervals of $3 \mathrm{~h}$, for both the HWM90 neutral wind model (June solstice, solid curves; December solstice, long-dashed curves) and the revised neutral wind model (June solstice, dot long-dashed curves; December solstice, short-dashed curves) latitude range $\pm 25^{\circ}$ are displayed in Fig. 7. This figure shows that the densities are higher at the December solstice than at the June solstice. The percentage differences of the densities are largest in $\mathrm{O}$ and smallest in $\mathrm{O}_{2}$, with $\mathrm{N}_{2}$ at an intermediate value. The largest

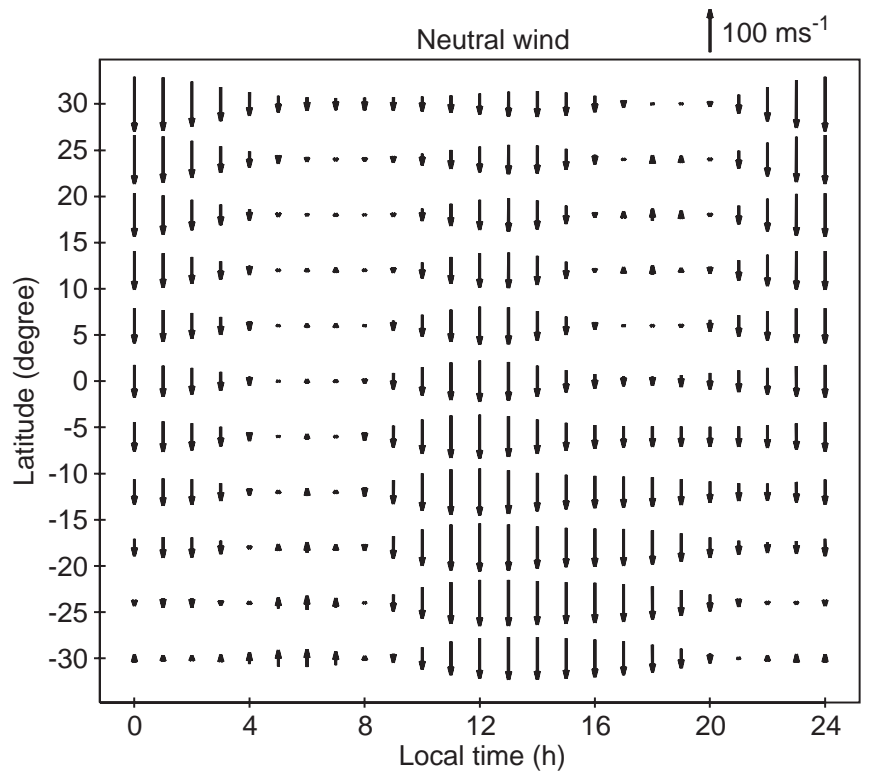

Fig. 5. Diurnal and latitudinal variations of the meridional neutral wind at $600 \mathrm{~km}$ altitude given by HWM90 for June solstice differences are about $25 \%, 15 \%$ and $8 \%$ for $\mathrm{O}, \mathrm{N}_{2}$ and $\mathrm{O}_{2}$, respectively. The average values for conjugate latitudes are not shown because the percentage differences of the densities, at each of the latitudes under consideration, are almost the same.

To investigate the effects of the neutral composition on the annual component in more detail, we have carried out two further sets of model calculations in which, except for the neutral densities, the model inputs are the same as those used to obtain the results for the short-dashed curves of Fig. 4 (December solstice with the revised neutral wind model). For the first set of additional calculations, the MSIS86 values for the densities of $\mathrm{O}, \mathrm{N}_{2}$ and $\mathrm{O}_{2}$ are multiplied by the factor 1.5 , for the second set, only the density of $\mathrm{O}$ is multiplied by 1.5. As shown by Fig. 8, the effect of increasing the densities of $\mathrm{O}, \mathrm{N}_{2}$ and $\mathrm{O}_{2}$ by $50 \%$ is to increase the electron density by about $40 \%$. On the other hand, a $50 \%$ increase in the $\mathrm{O}$ density alone gives rise to an increase in the electron density by about $60 \%$. These results arise since (1) an increase in the density of $\mathrm{O}$ will increase the production rate of the ions, which, in turn, will increase the electron density, and (2) an increase in the densities of $\mathrm{N}_{2}$ and $\mathrm{O}_{2}$ will increase the loss rate of ions, which, in turn, will decrease the electron density. The model results show that, to reproduce the observations that the electron densities at $600 \mathrm{~km}$ are about $100 \%$ higher at the December solstice than at the June solstice, the differences in the neutral gas densities between the two solstices should be more than $120 \%$ if 

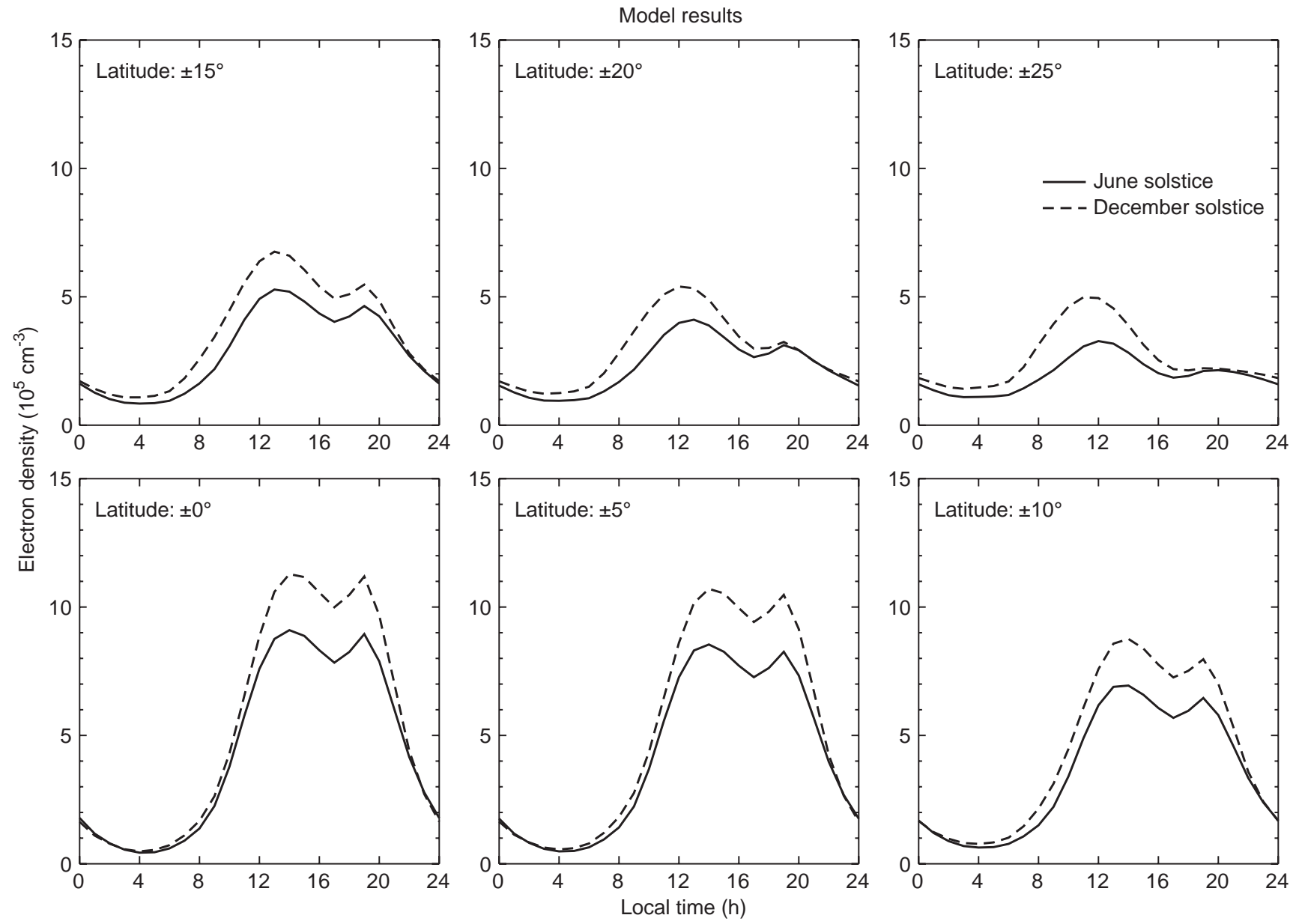

Fig. 6. Diurnal variations of the modelled electron density at latitude $0^{\circ}$, and the averages of the modelled electron densities at latitudes $\pm 5^{\circ}$, $\pm 10^{\circ}, \pm 15^{\circ}, \pm 20^{\circ}$ and $\pm 25^{\circ}$, for June solstice (solid curves) and December solstice (dashed curves)

both the atomic and molecular gas densities vary by the same percentage. However, if the atomic to molecular gas density ratio is higher at the December solstice than at the June solstice, a smaller difference in total neutral gas densities between the two solstices is required to reproduce the observations. In fact, the values predicted
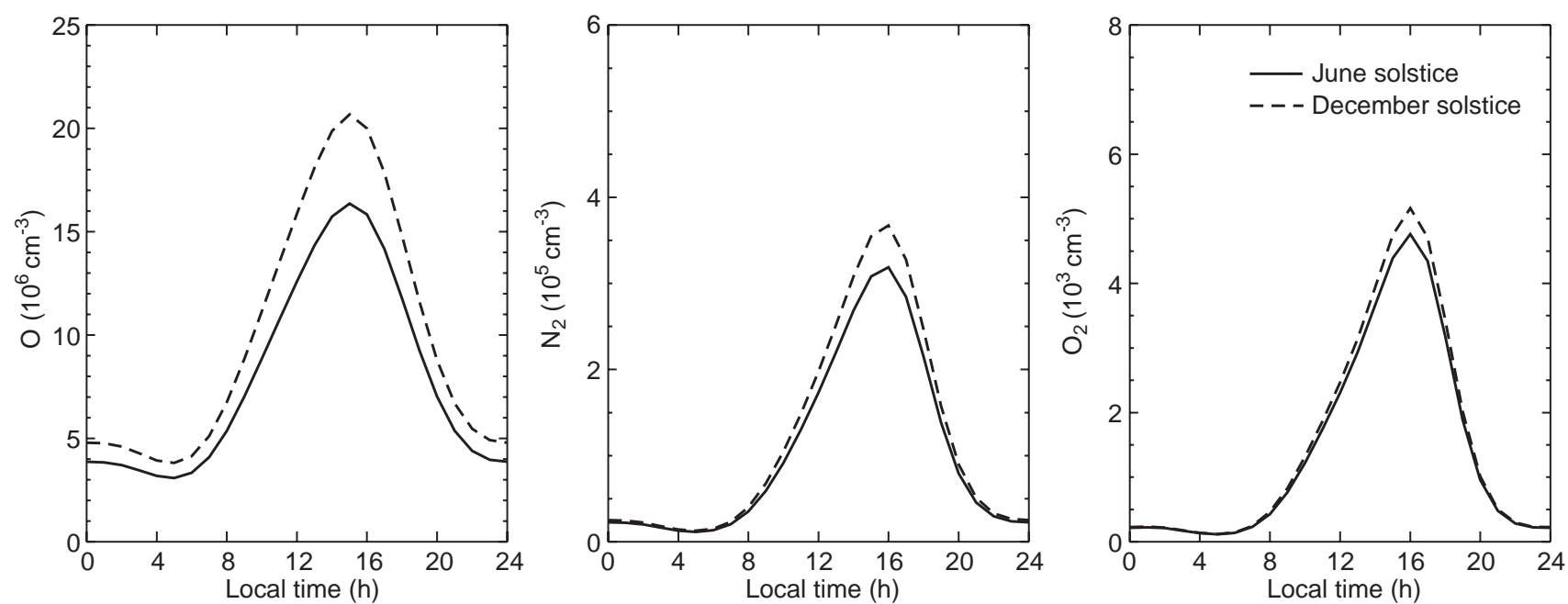

Fig. 7. Diurnal variations of the neutral gas $\left(\mathrm{O}, \mathrm{N}_{2}\right.$ and $\left.\mathrm{O}_{2}\right)$ densities averaged over the latitude range $\pm 25^{\circ}$ for June solstice (solid curves) and December solstice (dashed curves) 
by MSIS86 support our suggestion; MSIS86 predicts that the increase in density of the atomic gas, $\mathrm{O}$, between the June and December solstices is larger than that of the molecular gases, $\mathrm{N}_{2}$ and $\mathrm{O}_{2}$, see Fig. 7 .

The effects of the neutral wind and $\mathbf{E} \times \mathbf{B}$ drift velocities on the annual anomaly are investigated by comparing the present results with those obtained from calculations in which the daytime (08:00-18:00 LT) poleward wind velocity and the upward $\mathbf{E} \times \mathbf{B}$ drift velocity have been multiplied by the factor 2 . The results from these sets of calculations are displayed in Fig. 9. It is expected that the uncertainties in the average neutral wind and $\mathbf{E} \times \mathbf{B}$ drift velocities are within a factor of 2 . We have only changed the daytime values of the neutral wind and $\mathbf{E} \times \mathbf{B}$ drift velocities because the annual anomaly is mainly a daytime phenomenon. By comparing the solid curves with the dashed curves in Fig. 9 it can be seen that the effect of doubling the daytime neutral wind is to reduce the electron density at $600 \mathrm{~km}$; an increase in the poleward wind in both hemispheres depletes the topside ionosphere. The maximum reduction in electron density, which occurs in the afternoon sector when the polewind is strongest, is about $35 \%$. The model results suggest that the annual anomaly might be caused by the daytime poleward neutral winds, in both hemispheres, being stronger at the June solstice than at the December solstice. The main effect of a factor of 2 increase in the upward $\mathbf{E} \times \mathbf{B}$ drift velocity is to increase the electron density at $600 \mathrm{~km}$; an upward $\mathbf{E} \times \mathbf{B}$ drift moves plasma from the F region, where the electron density is high, to the topside ionosphere. The plasma fountain, a characteristic feature of the low-latitude ionosphere caused by upward $\mathbf{E} \times \mathbf{B}$ drift (Stening, 1992), gives rise to a decrease in the electron density at the equator and an increase at higher latitudes in the afternoon sector. The effects of the increase in $\mathbf{E} \times \mathbf{B}$ drift velocity lasts until midnight, although in the calculations the increase is switched off at 18:00 LT. The model results suggest that for the drift to contribute to the annual anomaly, the upward $\mathbf{E} \times \mathbf{B}$ drift velocity must be higher at the December solstice than at the June solstice.

\section{Discussions and conclusions}

The annual and seasonal variations of the topside ionosphere at low latitudes have been investigated using observations made by the Hinotori satellite. The observations show that the behaviours of both the annual and seasonal variations in the topside ionosphere are quite different to those in the $\mathrm{F}$ region. An outstanding feature in the topside ionosphere is that the annual anomaly is very large; the global average of the density at December solstice is about $100 \%$ higher than that at the June solstice compared with the $6 \%$ solar flux difference due to the changes in Sun-Earth distance. The fact that the annual anomaly is small in the $F$ region (Torr and Torr, 1973; Yonezawa, 1959, 1971) and large

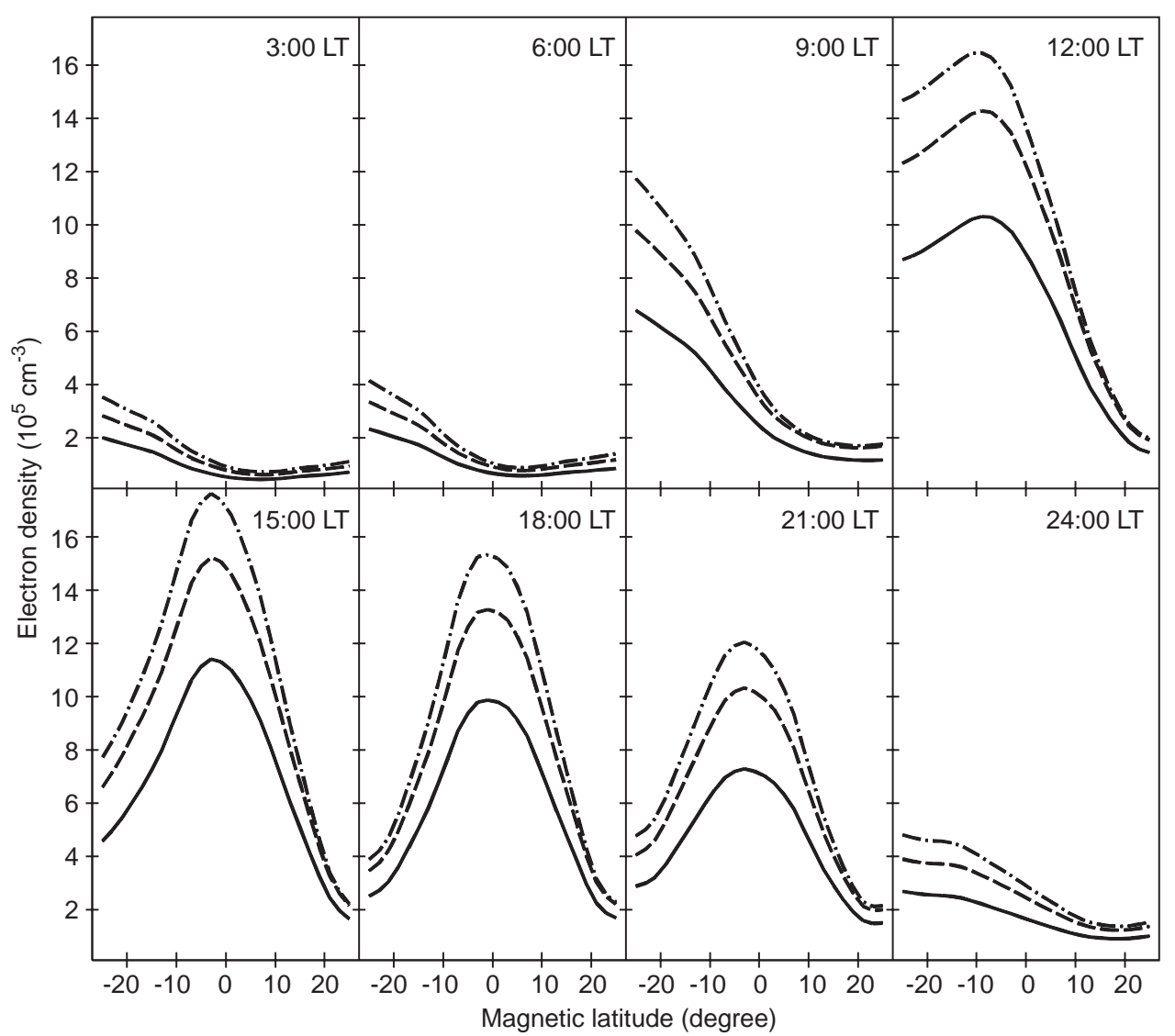

Fig. 8. Latitudinal variations of the modelled electron density, at intervals of $3 \mathrm{~h}$, for the December solstice. The solid curves are the same as the short-dashed curves of Fig. 4. The dashed curves and dotdashed curves are from the calculations in which the neutral densities, given by MSIS86, are multiplied by the factor 1.5 for $\mathrm{O}$, $\mathrm{O}_{2}$ and $\mathrm{N}_{2}$, and for $\mathrm{O}$ only, respectively. Other model inputs are the same as for the previous sets of calculations 


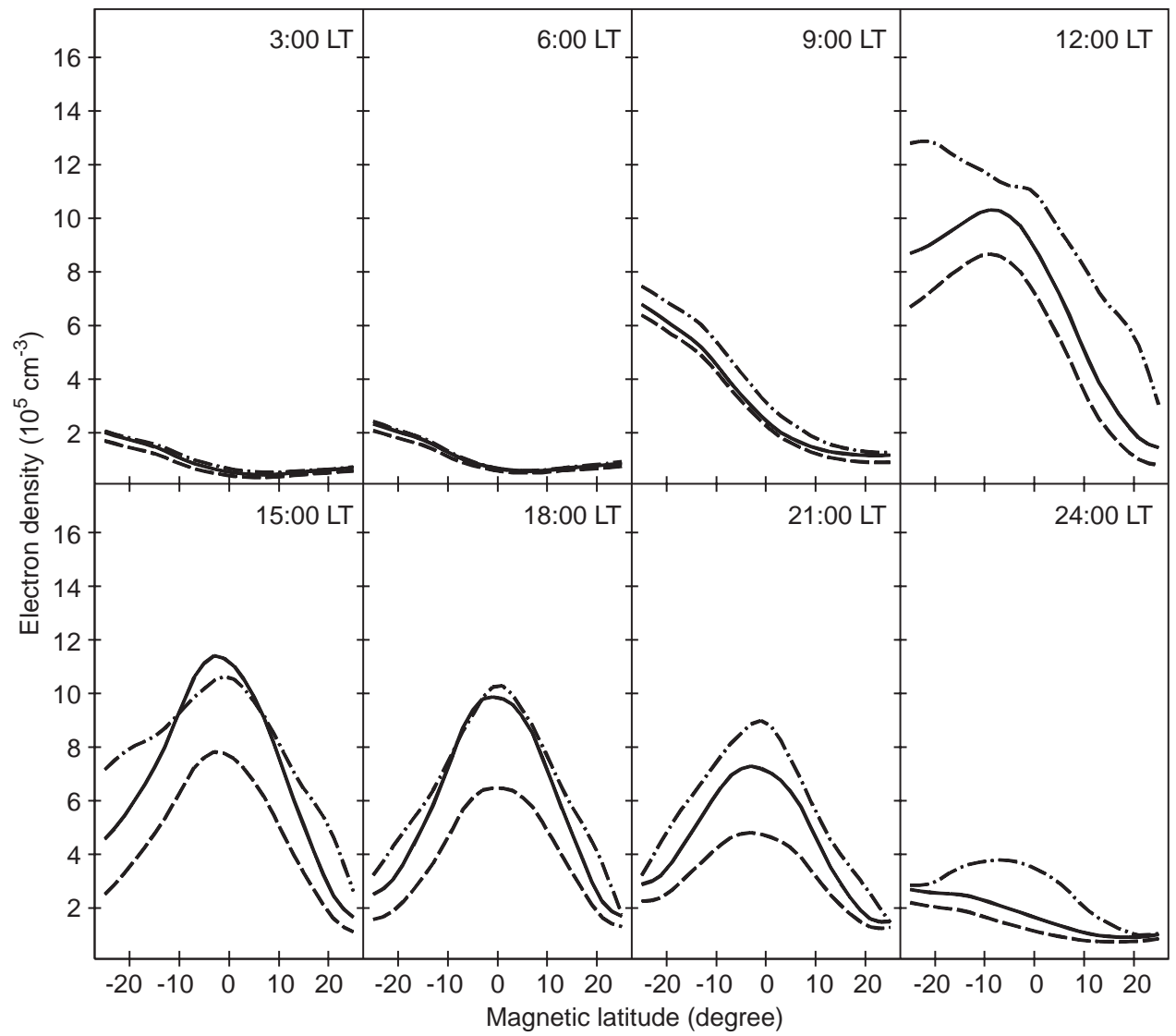

Fig. 9. Latitudinal variations of the modelled electron density, at intervals of $3 \mathrm{~h}$, for December solstice. The solid curves are the same as the short-dashed curves of Fig. 4. The dashed and dot-dashed curves are from the calculations in which the daytime (08:00-18:00 LT) poleward neutral wind and the upward $\mathbf{E} \times \mathbf{B}$ drift velocity are multiplied by the factor 2 , respectively. Other model inputs are the same as for the previous sets of calculations in the topside ionosphere suggests that the annual anomaly increases with altitude. Further support is provided by the fact that the annual anomaly predominates over the seasonal variations for TEC (Titheridge and Buonsanto, 1983) compared to the small annual component for NmF2.

Possible mechanisms for the observed annual and seasonal variations have been investigated by using values modelled by SUPIM. It has been found that while the seasonal variation is controlled by the neutral wind, the mechanism for the annual variation is rather complicated. Our model results suggest that an annual anomaly in the electron density occurs if the neutral gas densities and/or the atomic to molecular ratio is higher at the December solstice than at the June solstice. Values from the MSIS86 thermospheric model support this suggestion. However, if the values for the neutral gas densities given by MSIS86 are used in the model calculations then the differences in the modelled electron densities at the two solstices are only about $30 \%$ of the observed values. It should be borne in mind that most of the data used to construct the MSIS86 model have been collected from altitudes below $500 \mathrm{~km}$. Thus, the neutral gas densities predicted by MSIS 86 at $600 \mathrm{~km}$ altitude are based mainly on measurements made at lower altitudes; the predicted values might be somewhat different to the actual values.

The neutral wind might also play a role in the annual anomaly. Our model results suggest that if the daytime poleward winds are stronger at the June solstice than at the December solstice then the wind could contribute to the annual anomaly in the electron density. This suggestion has credibility because the observed electron densities are lower at the June solstice than at the December solstice and lower electron densities should give rise to larger neutral winds due to ion drag; ion drag acts to reduce the neutral wind velocity and is proportional to the electron density. Anderson and Roble (1974) indicate through model calculations that the variations in ion drag, due to variations in electron density, give rise to large variations in the neutral zonal wind velocity. It is expected that ion drag also significantly affects the meridional neutral wind velocity, although the effect of ion drag on the meridional wind is smaller than that on the zonal wind at low latitudes because the ions move more freely along the field lines than perpendicular to them. Also, changes in the zonal wind might change the neutral atmospheric circulation, which, in turn, would give rise to changes in the meridional wind.

The model results show that although a factor-of-2 increase in the daytime upward $\mathbf{E} \times \mathbf{B}$ drift velocity does not give rise to large changes in the electron density during daytime, the increase in the drift does give rise to increases in the electron density during the post-sunset period. On the other hand, observations of $\mathbf{E} \times \mathbf{B}$ drift velocity (Fejer et al., 1991, 1995) show that the difference in the average drift velocities of the two solstices 
during the morning to afternoon sector is much smaller than a factor of 2 and, in general, the upward drift velocity is larger at the June solstice than at the December solstice. The observed drift velocities also show an evening prereversal enhancement at the December solstice which is almost absent at the June solstice. Thus, the model calculations and the $\mathbf{E} \times \mathbf{B}$ drift velocity observations lead us to suggest that the $\mathbf{E} \times \mathbf{B}$ drift velocity might not contribute to, and might even slightly weaken, the annual anomaly in the morning to afternoon sector. However, our model results strongly suggest that the drift is responsible for the observed evening enhancement in electron density at the December solstice (see Fig. 2) that gives rise to the annual anomaly lasting to post-sunset period.

The model results discussed in the foregoing suggest that the interactions of thermosphere and ionosphere may strengthen the annual anomaly. To investigate the interactions of the ionosphere and thermosphere in a selfconsistent way, we have carried out model calculations using the Coupled Thermosphere Ionosphere Plasmasphere Model (CTIP, Millward et al., 1996b). Model results from CTIP show that with the same inputs of solar EUV flux and $\mathbf{E} \times \mathbf{B}$ drift for both June and December solstices the modelled average electron densities at $600 \mathrm{~km}$ are almost the same at the two solstices. Model calculations also show that a $6 \%$ increase in solar EUV fluxes gives rise to an increase in electron density by less than $10 \%$. These model results suggest that even if the interactions of the ionosphere and thermosphere are taken into account, differences in the electron densities at the two solstices, caused by the $6 \%$ difference in the EUV flux, should be much smaller than those seen in the observations. Thus, other energy sources, yet to be included in CTIP, may play an important role in creating large annual anomalies. It has been suggested (Torr and Torr, 1973) that the southern hemisphere may receive more energy than the northern hemisphere as a result of the asymmetry in the geomagnetic field. Because thermospheric circulation transports the neutral gases from summer hemisphere to winter hemisphere, the asymmetry of the energy input with respect to equator might give rise to that more energy is transported to the equatorial regions from southern hemisphere at December solstice than that from northern hemisphere at June solstice. Another possible energy source for the ionospheric annual anomaly are the tides in the mesosphere. There is observational evidence that the tidal intensity at December solstice is higher than at June solstice (Barlier et al., 1974). The energy of the tidal wave in the mesosphere can propagate upward to the thermosphere. Further observational evidence and modelling studies are required for understanding the relative importance of various energy sources on the ionospheric annual anomaly. Such work is beyond the objective of the present study.

Acknowledgements. The authors would like to thank H. Oya (Tohoku University) and T. Takahashi (Gumma University) for their efforts in organizing the electron density measurements onboard the Hinotori satellite. The work at Sheffield was supported by the Particle Physics and Astronomy Research Council (PPARC) under grants GR/K75422 and GR/L20238.

Topical Editor D. Alcaydé thanks two referees for their help in evaluating this paper.

\section{References}

Anderson, D. N., and R. G. Roble, The effect of vertical $\mathbf{E} \times \mathbf{B}$ ionospheric drifts on F-region neutral winds in the low-latitude thermosphere, J. Geophys. Res., 79, 5231-5236, 1974.

Bailey, G. J., and N. Balan, A low-latitude ionosphere-plasmasphere model, in STEP handbook on ionospheric models, Ed. R. W. Schunk, Utah State University, pp. 173-206, 1996.

Bailey, G. J., and R. Sellek, A mathematical model of the Earth's plasmasphere and its application in a study of $\mathrm{He}^{+}$, Ann. Geophysicae, 8, 171-190, 1990.

Bailey, G. J., R. Sellek, and Y. Rippeth, A modeling study of the equatorial topside ionosphere, Ann. Geophysicae, 11, 263-272, 1993.

Bailey, G. J., N. Balan, and Y. Z. Su, The Sheffield University plasmasphere ionosphere model - a review, J. Atmos. Terr. Phys., 59, 1541-1552, 1997.

Barlier, F., P. Bauer, C. Jaeck, G. Thuillier, and G. Kockarts, North-south asymmetries in the thermosphere during the last maximum of the solar cycle, J. Geophys. Res., 79, 5273-5285, 1974.

Buonsanto, M. J., Millstone Hill incoherent-scatter F-region observations during the disturbance of June 1991, J. Geophys. Res., 100, 5743-5755, 1995.

Fejer, B. G., F-region plasma drifts over Arecibo: solar cycle, seasonal, and magnetic activity effects, J. Geophys. Res., 98, 13645-13652, 1993.

Fejer, B. G., E. R. de Paula, S. A. González, and R. F. Woodman, Average vertical and zonal F-region plasma drifts over Jicamarca, J. Geophys. Res., 96, 13901-13906, 1991.

Fejer, B. G., E. R. de Paula, R. A. Heelis, and W. B. Hanson, Global equatorial ionospheric vertical plasma drifts measured by the AE-E satellite, J. Geophys. Res., 100, 5769-5776, 1995.

Hedin, A. E., MSIS-86 thermospheric model, J. Geophys. Res., 92, 4649-4662, 1987.

Hedin, A. E., M. A. Biondi, R. G. Burnside, G. Hernandez, R. M. Johnson, T. L. Killeen, C. Mazaudier, J. W. Meriwether, J. E. Salah, R. J. Sica, R. W. Smith, N. W. Spencer, V. B. Wickwar, and T. S. Virdi, Revised global model of thermosphere winds using satellite and ground-based observations, J. Geophys. Res., 96, 7657-7688, 1991 .

Millward, G. H., H. Rishbeth, T. J. Fuller-Rowell, A. D., Aylward, S. Quegan, and R. J. Moffett, Ionospheric F2-layer seasonal and semiannual variations, J. Geophys. Res., 101, 5149-5156, 1996 a.

Millward, G. H., R. J. Moffett, S. Quegan, and T. J. Fuller-Rowell, A coupled thermosphere-ionosphere-plasmasphere model (CTIP), in STEP handbook on ionospheric models, Ed. R. W. Schunk, Utah State University, pp. 239-279, 1996b.

Murphy, J. A., and R. A. Heelis, Implications of the relationship between electromagnetic drift components at mid and low latitudes, Planet. Space Sci., 34, 645-652, 1986.

Pingree, J. E., and B. G. Fejer, On the height variation of the equatorial F-region vertical plasma drifts, J. Geophys. Res., 92, 4763-4766, 1987.

Rishbeth, H., and C. S. G. K. Setty, The F layer at sunrise, J. Atmos. Terr. Phys., 20, 263-267, 1961.

Rüster, R., and J. W. King, Atmospheric composition changes and the F2-layer seasonal anomaly, J. Atmos. Terr. Phys., 35, 13171322, 1973.

Stening, R. J., Modelling the low-latitude F region, J. Atmos. Terr. Phys., 54, 1387-1412, 1992.

Su Y. Z., K.-I. Oyama, G. J. Bailey, T. Takahashi, and S. Watanabe, Comparison of satellite electron density and temperature measurements at low latitudes with a plasmasphereionosphere model, J. Geophys. Res., 100, 14591-14604, 1995. 
Su Y. Z., K.-I. Oyama, G. J. Bailey, S. Fukao, T. Takahashi, and H. Oya, Longitudinal variations of the topside ionosphere at low latitudes: satellite measurements and mathematical modellings, J. Geophys. Res., 101, 17191-17205, 1996.

Su Y. Z., S. Fukao, and G. J. Bailey, Modeling studies of the middle and upper atmosphere radar observations of the ionospheric F layer, J. Geophys. Res., 102, 319-327, 1997.

Titheridge, J. E., and M. J. Buonsanto, Annual variations in the electron content and height of the $\mathrm{F}$ layer in the northern and southern hemispheres, related to neutral composition, J. Atmos. Terr. Phys., 45, 683-696, 1983.

Tobiska, W. K., Revised solar extreme ultraviolet flux model, J. Atmos. Terr. Phys., 53, 1005-1018, 1991.

Torr, M. R., and D. G. Torr, The seasonal behaviour of the F2-layer of the ionosphere, J. Atmos. Terr. Phys., 35, 2237-2251, 1973.
Watanabe, S., and H. Oya, Occurrence characteristics of low-latitude ionosphere irregularities observed by impedance probe on board the Hinotori satellite, J. Geomagn. Geoelectr., 38, 125-149, 1986.

Yonezawa, T., On the seasonal and non-seasonal annual variations and the semi-annual variation in the noon and midnight densities of the F2-layer in middle latitudes, II, J. Radio Res. Lab. Jpn., 6, 651-660, 1959.

Yonezawa, T., The solar-activity and latitudinal characteristics of the seasonal, non-seasonal and semi-annual variations in the peak electron densities of the F2-layer at noon and at midnight in middle and low latitudes, J. Atmos. Terr. Phys., 33, 889-907, 1971. 